\title{
The MEP pathway in Babesia orientalis apicoplast, a potential target for anti- babesiosis drug development
}

Lan He $\mathrm{e}^{1,2,3}$, Pei He $\mathrm{1}^{1,2}$, Xiaoying Luo ${ }^{1,2}$, Muxiao Li ${ }^{1,2}$, Long Yu ${ }^{1,2}$, Jiaying Guo ${ }^{1,2}$, Xueyan Zhan ${ }^{1,2}$, Guan Zhu ${ }^{4}$ and Junlong Zhao ${ }^{1,2,3^{*}}$

\begin{abstract}
Background: The apicomplexan parasite Babesia orientalis, the causative agent of water buffalo babesiosis in China, is widespread in central and south China, resulting in a huge economic loss annually. Currently, there is no effective vaccine or drug against this disease. Babesia bovis and Plasmodium falciparum were reported to possess an apicoplast which contains the methylerythritol phosphate (MEP) pathway inhibitable by fosmidomycin, suggesting that the pathway could serve as a drug target for screening new drugs. However, it remains unknown in B. orientalis.

Methods: Primers were designed according to the seven MEP pathway genes of Babesia microti and Babesia bovis. The genes were cloned, sequenced and analyzed. The open reading frames (ORFs) of the first two enzyme genes, 1-deoxyD-xylulose 5-phosphate synthase (BoDXS) and 1-Deoxy-D-xylulose 5-phosphate reductoisomerase (BoDXR), were cloned into the pET-32a expression vector and expressed as a Trx-tag fusion protein. Rabbit anti-rBoDXS and rabbit anti-rBoDXR antibodies were generated. Western blot was performed to identify the native proteins of BoDXS and BoDXR in B. orientalis. Fosmidomycin and geranylgeraniol were used for inhibition assay and rescue assay, respectively, in the in vitro cultivation of $B$. orientalis.
\end{abstract}

Results: The seven enzyme genes of the $B$. orientalis MEP pathway (DXS, DXR, IspD, IspE, IspF, IspG and IspH) were cloned and sequenced, with a full length of 2094, 1554, 1344, 1521, 654, 1932 and 1056 bp, respectively. BoDXS and BoDXR were expressed as Trx-tag fusion proteins, with a size of 95 and $67 \mathrm{kDa}$, respectively. Western blot identified a $77 \mathrm{kDa}$ band for the native BoDXS and a $49 \mathrm{kDa}$ band for the native BoDXR. The drug assay results showed that fosmidomycin could inhibit the growth of $B$. orientalis, and geranylgeraniol could reverse the effect of fosmidomycin.

Conclusions: Babesia orientalis has the isoprenoid biosynthesis pathway, which could be a potential drug target for controlling and curing babesiosis. Considering the high price and instability of fosmidomycin, further studies should focus on the screening of stable and cheap drugs.

Keywords: Babesia orientalis, Apicoplast, Isoprenoid, Methylerythritol phosphate (MEP) pathway, 1-deoxy-Dxylulose 5-phosphate reductoisomerase (DXR), Fosmidomycin

\footnotetext{
* Correspondence: zhaojunlong@mail.hzau.edu.cn

'State Key Laboratory of Agricultural Microbiology, College of Veterinary Medicine, Huazhong Agricultural University, Wuhan 430070, Hubei, People's Republic of China

${ }^{2}$ Key Laboratory for Development of Veterinary Diagnostic Products, Ministry of Agriculture, Huazhong Agricultural University, Wuhan 430070, Hubei, People's Republic of China

Full list of author information is available at the end of the article
}

(c) The Author(s). 2018 Open Access This article is distributed under the terms of the Creative Commons Attribution 4.0 International License (http://creativecommons.org/licenses/by/4.0/), which permits unrestricted use, distribution, and reproduction in any medium, provided you give appropriate credit to the original author(s) and the source, provide a link to the Creative Commons license, and indicate if changes were made. The Creative Commons Public Domain Dedication waiver (http://creativecommons.org/publicdomain/zero/1.0/) applies to the data made available in this article, unless otherwise stated. 


\section{Background}

Many members of the Apicomplexa, including Plasmodium, Toxoplasma, Eimeria, Babesia and Theileria, contain a unique, relic, non-photosynthetic plastid-like organelle termed apicoplast [1]. The apicoplast is a semi-autonomous organelle which normally contains a $35 \mathrm{kbp}$ circular genome. There are three pathways in the apicoplast of Plasmodium and Toxoplasma, including the methylerythritol phosphate (MEP) pathway for isoprenoid biosynthesis, FAS pathway and heme operate. In B. bovis and Babesia microti, the isoprenoid biosynthesis MEP pathway is the only metabolic pathway in the apicoplast $[2,3]$. Isopentenyl diphosphate (IPP) and dimethylallyl diphosphate (DMAPP) are two precursors to construct all the isoprenoids which are important for organisms. Isoprenoids comprise a large group of diverse intracellular metabolites with multiple cellular functions, including roles in membrane structure, cellular respiration and cell signaling [4].

In nature, there are two distinct biosynthetic routes to produce IPP and DMAPP: methylerythritol phosphate (MEP) pathway and a modified mevalonate (MVA) pathway. The mevalonic acid (MVA) pathway was thought to be the only IPP and DMAPP biosynthetic pathway for decades [5]. Mammals, including humans, use the MVA pathway that initiates from mevalonate, whilst eubacteria, cyanobacteria, plant chloroplasts and apicomplexan parasites exploit the methylerythritol phosphate (MEP) pathway that starts from pyruvate [6].

The MEP pathway is essential in eubacteria and protozoan but absent in humans and animals which could be the hosts of eubacteria and protozoan. The MEP pathway was discovered in the 1990s [7]. In this pathway, the reaction of all the steps are catalyzed by seven different enzymes, including 1-deoxy-D-xylulose 5-phosphate synthase (DXS), 1-deoxy-D-xylulose 5-phosphate reductoisomerase (DXR or IspC), 4-diphosphocytidyl-2C-methyl-D-erythritol cytidylyltransferase (IspD), 4-diphosphocytidyl-2C-methyl-D-er ythritol kinase (IspE), 2C-methyl-D-erythritol-2, 4-cyclodip hosphate synthase (IspF), 1-hydroxy-2-methyl-2-(E)-buteny 1-4-diphosphate synthase (IspG) and 1-hydroxy-2-methyl2-(E)-butenyl-4-diphosphate reductase (IspH) [6]. The second enzyme DXR catalyzes step II of the conversion of 1-deoxy-D-xylulose 5-phosphate (DOXP) to 2-C-methyl-D-erythritol 4-phosphate (MEP), which is a target for fosmidomycin [8]. Fosmidomycin, a broad-spectrum antimicrobial agent showing the clinical promise of antimalarial drug, inhibits the isoprenoid biosynthesis by suppressing the activity of $P$. falciparum DXR, a rate-limiting enzyme of the MEP pathway, and the parasites will die for lack of isoprenoids (by blocking the conversion of DOXP to 2-C-methyl-D-erythritol 4-phosphate). Fosmidomycin is currently under Phase II clinical trials in combination with clindamycin and piperaquine against malaria [8-10].
Babesia spp. are tick-borne intraerythrocytic protozoans and cause babesiosis globally. Babesia orientalis, a recently identified species epidemic in China, causes water buffalo babesiosis. The parasite is transovarially transmitted by Rhipicephalus haemaphysaloides which is the only reported vector of B. orientalis, and water buffalo is the only reported host. As a member of the family Babesiidae, $B$. orientalis has a similar life-cycle with other babesiid species, including a sexual stage within $R$. haemaphysaloides, followed by an asexual stage in water buffalo RBC. Babesiosis has become one of the most important water buffalo diseases in central and south China [11]. However, there is no commercial vaccine or drug available to cure the disease. While the MEP pathway plays a critical role in the parasites and is reported to be a potential drug target, little information is available on the MEP pathway in $B$. orientalis. In this study, we proved that $B$. orientalis has the MEP pathway, and fosmidomycin can inhibit the growth of $B$. orientalis. The results demonstrate that the MEP pathway could serve as a potential target for screening anti-babesiosis drugs.

\section{Methods}

Parasites

Babesia orientalis (Wuhan strain) was previously isolated from Wuhan city, Hubei Province, China, and preserved in liquid nitrogen in the State Key Laboratory of Agricultural Microbiology, Huazhong Agricultural University, China.

Three one-year-old water buffaloes were purchased from a Babesia-free area. The buffaloes were tested by Giemsa staining and loop-mediated isothermal amplification (LAMP) to confirm that they were Babesia free/ negative [12]. One water buffalo was used as a negative control. The other two water buffaloes were splenectomized 2 weeks before infection, and subcutaneously injected with $4 \mathrm{ml}$ of $B$. orientalis positive blood (1\% parasitemia). Blood was collected when the parasitemia of the infected buffaloes reached 3\%.

\section{DNA extraction}

Blood from the jugular vein of experimentally infected water buffaloes was collected in tubes containing EDTA (BD Vacutainer, USA). Genomic DNA was extracted from $200 \mu \mathrm{l}$ of $B$. orientalis-infected blood using the QIAamp DNA Mini Kit (Qiagen, Shanghai, China) according to the manufacturer's instructions. The DNA samples were used immediately or stored at $-20^{\circ} \mathrm{C}$ until use.

\section{RNA extraction and CDNA synthesis}

Total RNA was extracted from the purified $B$. orientalis merozoites by using the TRIZOL reagent (Invitrogen, Shanghai, China) and treated with RNase-free DNaseI (TaKaRa, Dalian, China). RNA concentration was measured by Nanodrop 2000. The cDNA was prepared from 
$1 \mu \mathrm{g}$ of the total RNA using PrimeScript ${ }^{\mathrm{TM}}$ RT reagent Kit with gDNA eraser (TaKaRa, Dalian, China) according to the manufacturer's instructions.

\section{Cloning of the seven enzyme genes of the MEP pathway} Primer pairs for the seven enzyme genes of the $B$. orientalis MEP pathway were designed based on the gene sequences of B. microti and B. bovis (Table 1). Complete sequences of the seven genes were amplified by conventional PCR from genomic DNA and cDNA separately. PCR products were purified and ligated into the cloning vector pDM19-T (TaKaRa, Dalian, China). Three positive colonies of each gene were sent for sequencing. The nucleotide sequences of the seven genes were submitted to GenBank.

\section{Sequence analysis}

The amino acid sequence of BoDXR was aligned with the amino acid sequences from other organisms, including $B$. bovis, P. falciparum, Arabidopsis thaliana and Escherichia coli. The alignment was performed by using the program MAFFT v7 [13] and then edited in BioEdit. Phylogenetic analysis of the aligned sequences was conducted using the neighbor-joining algorithm in MEGA 6 [14].

\section{Expression and identification of BoDXS and BoDXR}

The open reading frame (ORF) of BoDXS was cloned into the pET-32a vector and expressed in E. coli BL21 (DE3). The recombinant protein was purified by affinity purification using a Ni-charged NTA column. Antisera against BoDXS were raised in rabbits.

In order to identify the native DXS in $B$. orientalis, the lysates of erythrocytes prepared from $B$. orientalis and Babesia-free water buffaloes (the negative control) $(0.5 \mathrm{mg}$

\begin{tabular}{|c|c|c|}
\hline Primer & Sequence $\left(5^{\prime}-3^{\prime}\right)$ & Amplicon size (bp) \\
\hline DXS-F & ATGGAGTTGTGTTGTAATC & 2094 \\
\hline DXS-R & TTAGGATGCAAGGAATTGG & \\
\hline DXR-F & ATGAATGCAGCAGTGAGTIITTATG & gDNA 1554 \\
\hline DXR-R & TTAGTATGTGAAGCATTAATATATGTGATAG & cDNA 1371 \\
\hline IspD-F & ATGTCTGTCTGGTTGGTGCAATG & 1344 \\
\hline IspD-R & TCACGGGAAGTATACCTCTITAAG & \\
\hline IspE-F & ATGAGGCATCTCCATGTGTG & 1521 \\
\hline IspE-R & TCAAGAGATTAGGTCTGGAAGAAG & \\
\hline IspF-F & ATGTTACTACGTTATCTITCTATAACAG & 654 \\
\hline IspF-F & TTATAAAGGATATTACTTGAGCTTC & \\
\hline IspG-F & ATGACATCGTCAGACACCTTTG & 1932 \\
\hline IspG-R & TCACATGCTTCCACATCTGG & \\
\hline $\mathrm{IspH}-\mathrm{F}$ & ATGGAGGAAAGACCGTTTACC & 1056 \\
\hline IspH-R & TTATGTAGTITCCAGATTCCTATAATCG & \\
\hline
\end{tabular}

per lane) were separated by $12 \%$ SDS-PAGE, transferred onto nitrocellulose membrane (Millipore, Shanghai, China) and probed separately with rabbit serum raised against rBoDXS and pre-immune serum (1:100 dilution). The membranes were washed with PBST, and then incubated with the secondary antibody (1:5000, goat anti-rabbit IgG HRP, Beyotime, Shanghai, China). The expression and identification of BoDXR were conducted as previously described for BoDXS.

\section{Babesia orientalis in vitro cultivation and growth inhibition assay}

Briefly, B. orientalis in the water buffalo erythrocytes was cultured in M199 media (Gibco Life Technologies, Shanghai, China) supplemented with 1\% Albuman I (Gibco Life Technologies), 2\% HB101 (Irvine Scientific, Shanghai, China), 1\% L-glutamine (ATLANTA Biologicals, Shanghai, China), 2\% Antibiotic/Antimycotic 100× (Corning, Shanghai, China) and $20 \%$ healthy water buffalo serum with $10 \%$ hematocrit $(\mathrm{HCT})$ at $37{ }^{\circ} \mathrm{C}$ in a microaerophilous stationary phase $\left(5 \% \mathrm{CO}_{2}, 2 \% \mathrm{O}_{2}, 93 \% \mathrm{~N}_{2}\right)$. The cultures were sub-cultured every three days when the parasitemia reached $2-3 \%$.

Drug stock solutions of fosmidomycin (Sigma-Aldrich, Shanghai, Chain) and diminazene aceturate (Sigma-Aldrich, Shanghai, Chain) were prepared in sterile water. Geranylgeraniol (Sigma-Aldrich, Shanghai, Chain) stocks were prepared in $100 \%$ ethanol. For the growth inhibition assay, B. orientalis cultures (total volume, $150 \mu \mathrm{l} /$ well) were grown in 96-well flat-bottomed plates containing fosmidomycin in culture media. In the rescue experiments, the parasites were divided into five separate treatment groups $(5 \mu \mathrm{M}$ fosmidomycin, $5 \mu \mathrm{M}$ fosmidomycin plus $5 \mu \mathrm{M}$ geranylgeraniol, $5 \mu \mathrm{M}$ geranylgeraniol plus $0.4 \mu \mathrm{M}$ diminazene aceturate, $0.4 \mu \mathrm{M}$ diminazene aceturate, and $0.1 \%$ ethanol). Diminazene aceturate was used as a positive control drug and ethanol was used as a blank control.

During the $72 \mathrm{~h}$ incubation of the plates, three smears were prepared from each well and the parasitemias were determined every $24 \mathrm{~h}$ by microscopy. Thin blood smears were air-dried, fixed with methanol, and stained with Giemsa (Sigma-Aldrich, Shanghai, China) according to the manufacturer's instructions. The cultures were prepared in triplicate. The data were analyzed by GraphPad Prism 5 by one-way analysis of variance (ANOVA) followed by Turkey's multiple comparison test. Error bars represent standard deviations.

\section{Results and discussion}

\section{Characterization of the enzyme genes}

According to the reported genome sequences of $B$. bovis and $B$. microti, the genomes encode all the seven components of the isoprenoid pathway, suggesting that the 
parasites have the MEP pathway [3, 15]. In this study, the seven enzyme genes of the $B$. orientalis MEP pathway were amplified from both gDNA and cDNA and sequenced. The full lengths of DXS, DXR, IspD, IspE, IspF, IspG and IspH were 2094, 1554, 1344, 1521, 654, 1932 and 1056 bp, respectively (Fig. 1). These results indicated that all seven genes of the MEP pathway exist in the genome of $B$. orientalis. The nucleotide sequences of the DXS, DXR, IspD, IspE, IspF, IspG and IspH were submitted to the GenBank database under the accession numbers MH429606, MH429607, MH429608, MH448076, MH429609, MH429610 and MH429611, respectively.

BoDXS is intronless, encoding 667 aa with a predicted size of $77 \mathrm{kDa}$. BoDXR contains a $184 \mathrm{bp}$ intron because the sequence obtained from cDNA is shorter than that of gDNA. It is the only intron gene in $B$. orientalis MEP pathway. BoDXR ORF is 1371 bp long, encoding 456 aa with a predicted size of $49 \mathrm{kDa}$. The amino acid sequence of BoDXR was aligned with the sequences of B. bovis (XP_001612194), Plasmodium falciparum (AAD03739), Arabidopsis thaliana (NP_201085) and Escherichia coli (EGT67209) (Fig. 2). The results indicated that DXR is highly conserved between these organisms and most close to B. bovis. For a better understanding of BoDXR and its homologs from related apicomplexans, a neighbor-joining tree was constructed. The results showed that BoDXR was most closely related to DXR from B. bovis. Sequence analysis indicated that BoDXR contains a NADB Rossmann superfamily domain and two DOXP redisom $C$ superfamily domains. The NADB domain is found in numerous dehydrogenases of metabolic pathways, and it is responsible for specifically binding a substrate and catalyzing the enzyme reaction. The DOXP redisom $\mathrm{C}$ domain is the DXR C-terminus, which is also found in the DXR enzyme of bacteria and plants. It catalyzes the formation of 2-C-methyl-D-erythritol 4-phosphate (MEP) from 1-deoxy-D-xylulose-5-phosphate (DOXP) in the presence of NADPH.

\section{Characterization of native BoDXS and BoDXR}

The initial enzyme DXS and the rate-limited enzyme DXR are the most important enzymes of the MEP pathway. 1-deoxy-D-xylulose 5-phosphate (DOXP), the product of DXS, also participates in thiamine (vitamin B1) biosynthesis and/or pyridoxine (B6) synthesis [16, 17], indicating that DXS is not a committed member of the MEP pathway. However, DXS is the first enzyme of the MEP pathway, and it catalyzes pyruvate and glyceraldehydes-3-phosphate to DOXP in the apicoplast of apicomplexan parasites. The second enzyme DXR, which is considered as the first committed enzyme of the MEP pathway, synthesizes 2-C-methyl-D-erythritol 4-phosphate (MEP) by intramolecular rearrangement and reduction of DOXP [17]. For biochemical characterization of BoDXS and BoDXR enzymes, the corresponding genes were over-expressed in E. coli and purified by Ni-NTA affinity chromatography. The purified rBoDXS and rBoDXR revealed the two bands of 95 and $67 \mathrm{kDa}$ on $10 \%$ SDS-PAGE, including an additional $18 \mathrm{kDa}$ Trx-tag (Fig. 3). Antibodies were generated in rabbit for detecting the native BoDXS and BoDXR. After the reaction of the rabbit anti-rBoDXS serum and rabbit anti-rBoDXR serum with $B$. orientalis lysates, the specific bands of 77 and 49 $\mathrm{kDa}$ were yielded, but not in the reaction with the lysates of the healthy bovine erythrocytes or pre-immune rabbit serum (Fig. 4). The results indicated that B. orientalis contains the native enzymes DXS and DXR.

\section{Fosmidomycin inhibits the growth of $B$. orientalis}

The isoprenoid biosynthesis pathway generates isoprenoid precursors, and it is a promising chemotherapeutic target due to the difference between mammals and apicomplexan parasites in the seven enzymes of this pathway [8]. To date, the best characterized inhibitor of the MEP pathway in parasites is the phosphonic acid antibiotic fosmidomycin, a potential inhibitor of DXR by

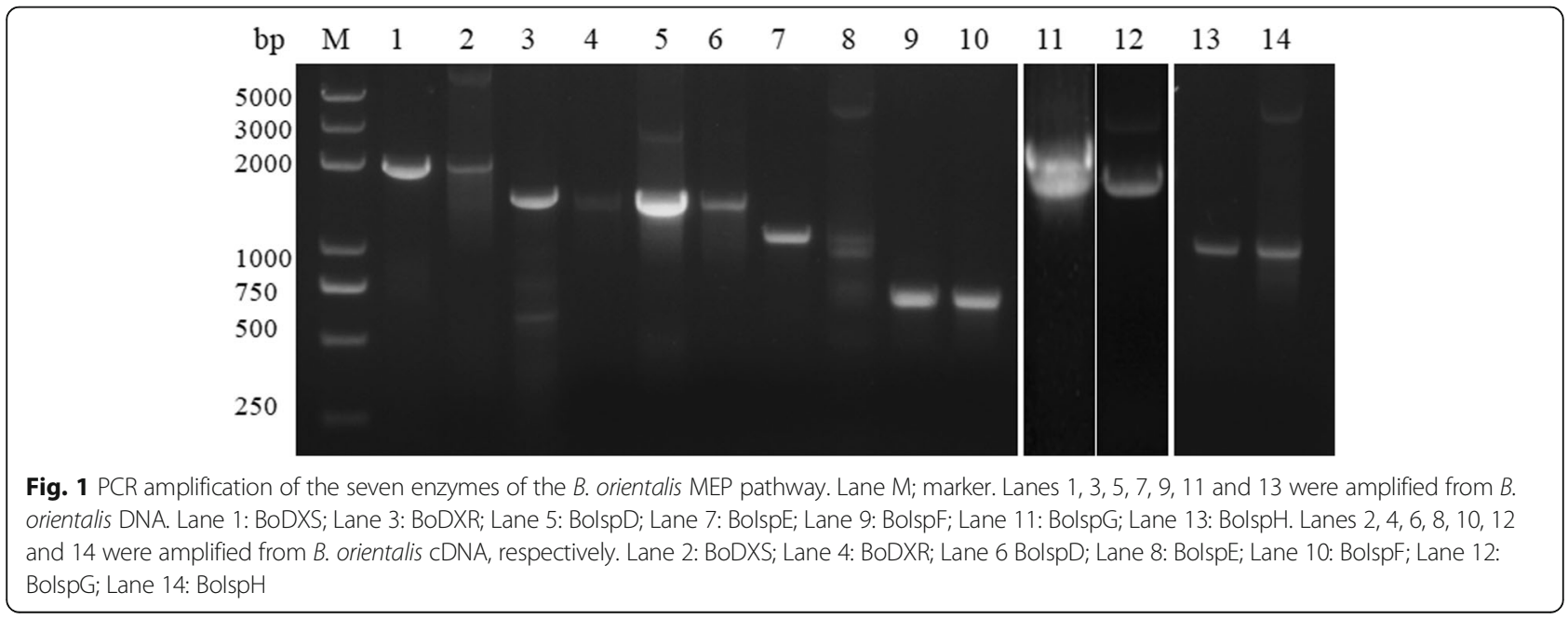




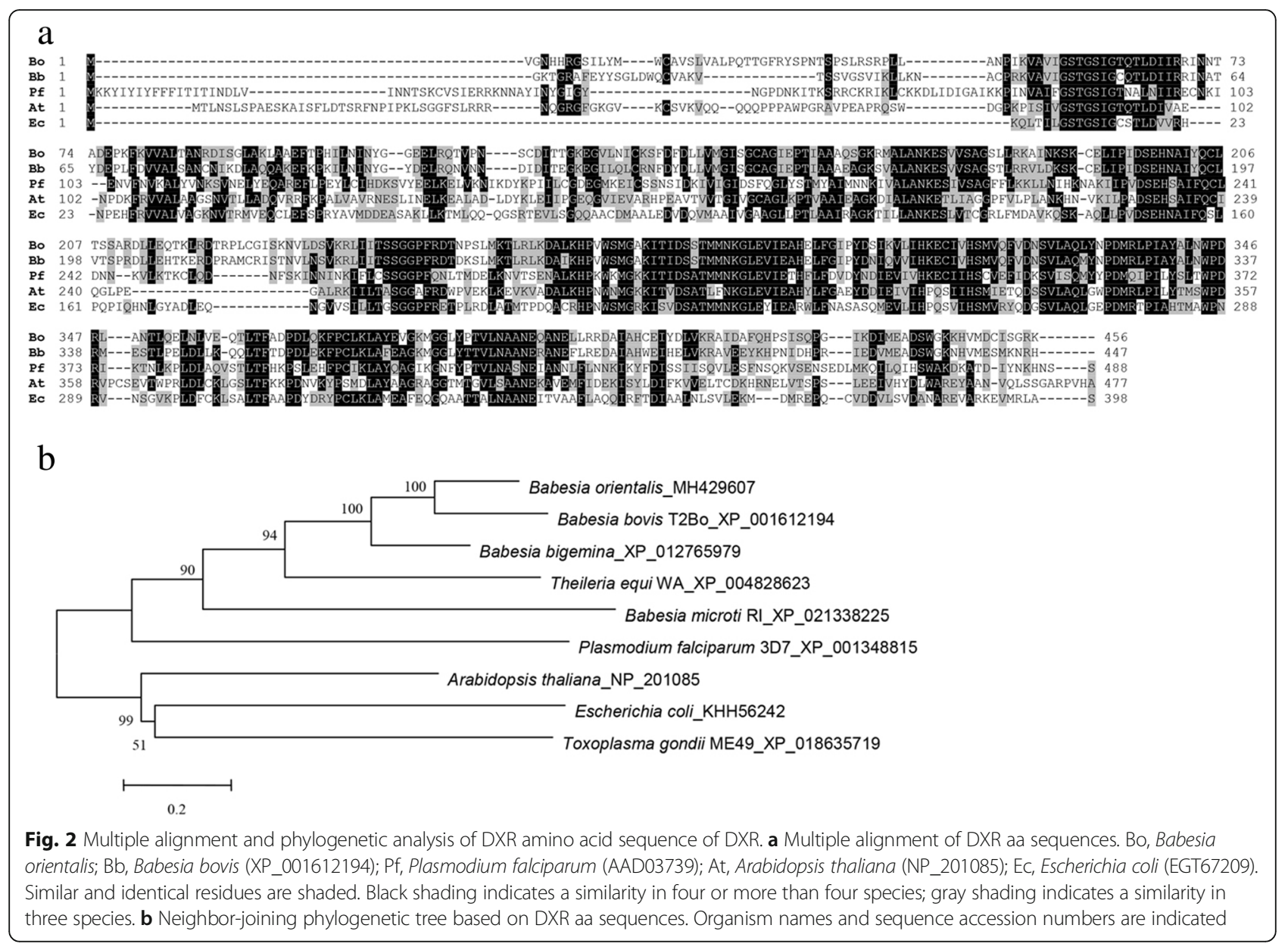

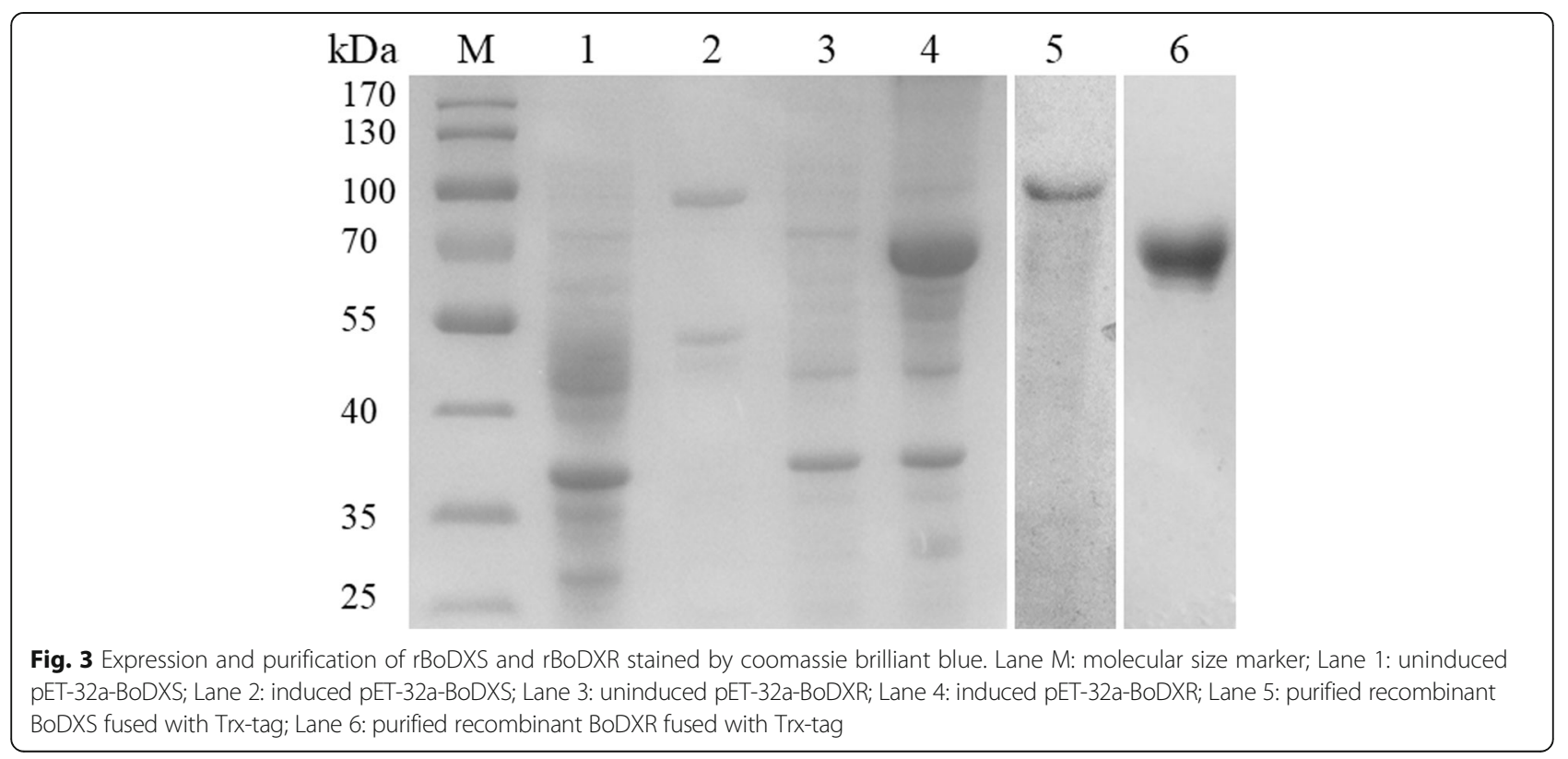


a

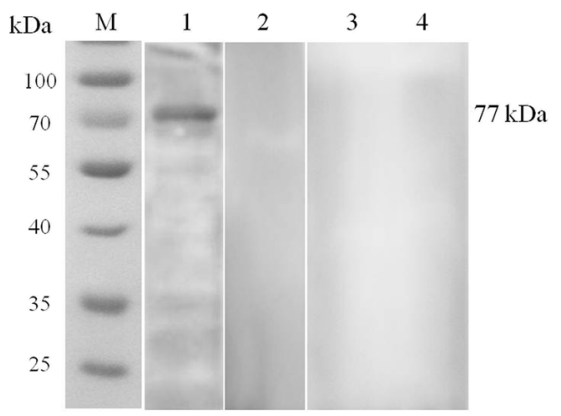

b

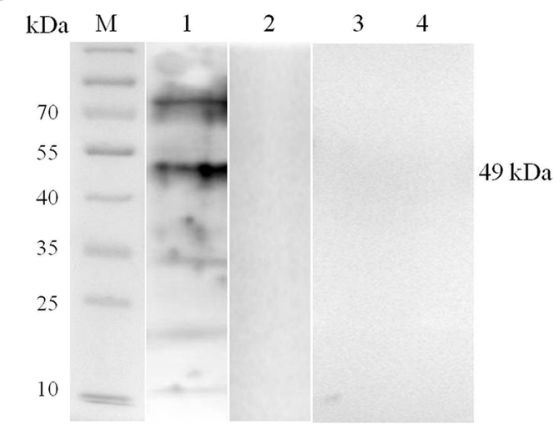

Fig. 4 Identification of native BoDXS and BoDXR enzymes in B. orientalis merozoite lysates. a Identification of native BoDXS. Lysates of B. orientalisinfected water buffalo erythrocytes and lysates of uninfected water buffalo erythrocytes were both probed with the serum from rabbit immunized with rBoDXS (Lane 1 and Lane 3, respectively) and the pre-immune rabbit serum (Lane 2 and Lane 4, respectively). $\mathbf{b}$ Identification of native BoDXR. Lysates of $B$. orientalis-infected water buffalo erythrocytes and lysates of uninfected water buffalo erythrocytes were both probed with the serum from rabbit immunized with rBoDXR (Lane 1 and Lane 3, respectively) and the pre-immune rabbit serum (Lane 2 and Lane 4, respectively)

blocking the conversion of DOXP to 2-C-methyl-D-erythritol 4-phosphate [18]. Fosmidomycin is under Phase II clinical trials as a potential antimalarial chemotherapeutic agent in combination with clindamycin and piperaquine $[8,10]$. The parasite Babesia is similar to Plasmodium in many aspects, and fosmidomycin was reported to be able to inhibit the intracellular growth of $B$. divergens at pharmacologically relevant concentrations [19]. However, there is no report about the inhibition of $B$. orientalis growth by fosmidomycin.

In the present study, $B$. orientalis was cultured and treated separately with $5 \mu \mathrm{M}$ fosmidomycin, $5 \mu \mathrm{M}$ fosmidomycin plus $5 \mu \mathrm{M}$ geranylgeraniol, $5 \mu \mathrm{M}$ geranylgeraniol plus $0.4 \quad \mu \mathrm{M}$ diminazene aceturate, $0.4 \mu \mathrm{M}$ diminazene aceturate, and $0.1 \%$ ethanol. Diminazene aceturate was used as a positive drug control. Ethanol was used as a control because geranylgeraniol was soluble in ethanol (treated concentration $0.1 \%$ ). The parasitemias were examined by Giemsa staining and light microscopy every $24 \mathrm{~h}$. The results indicated that fosmidomycin was effective in inhibition of $B$. orientalis growth after $24 \mathrm{~h}$ (Fig. 5), which is consistent with previous studies in Babesia bovis and Plasmodium falciparum $[8,15]$. We found that both fosmidomycin and diminazene aceturate exhibited anti-babesiosis activity at 48 $\mathrm{h}$ and $72 \mathrm{~h}$. The relative growth of fosmidomycin and negative controls were significant different at $48 \mathrm{~h}$ (ANOVA, $\left.F_{(3,8)}=23.07, P=0.0015\right)$ and $72 \mathrm{~h}$ treatment (ANOVA, $\left.F_{(3,8)}=73.21, P<0.0001\right)$. However, there was no notable difference between fosmidomycin plus geranylgeraniol and control groups (both negative control and $0.1 \%$ ethanol) (Fig. 6). The effects of fosmidomycin were reversed by medium supplemented with a downstream isoprenol geranylgeraniol, confirming that they are specific to isoprenoid blockade.

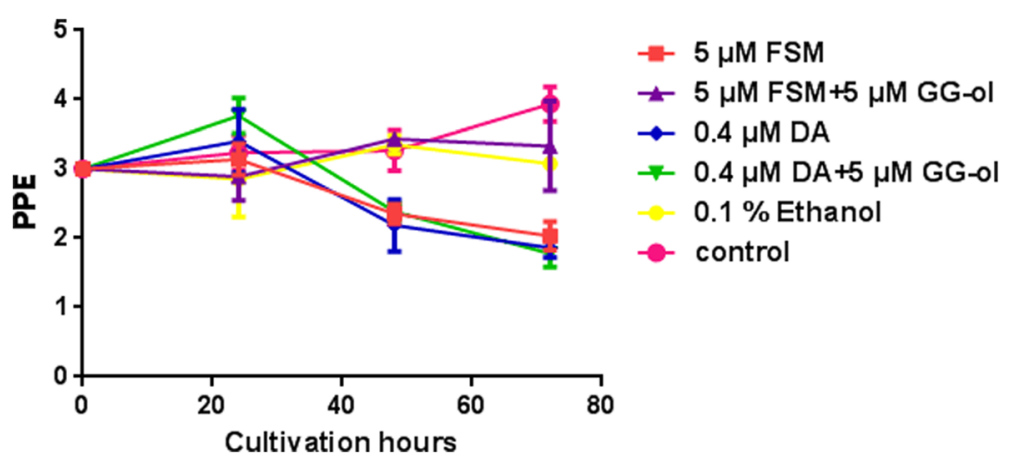

Fig. 5 Fosmidomycin and geranylgeraniol treated parasites arrest during in vitro cultivation of B. orientalis. The parasites were treated separately with fosmidomycin (FSM), fosmidomycin plus geranylgeraniol (GG-ol), diminazene aceturate (DA), diminazene aceturate plus geranylgeraniol, and ethanol (control). Negative control without any treatment was also included. The parasitemias were determined by Giemsa staining every $24 \mathrm{~h}$. PPE, percent parasitized erythrocytes 


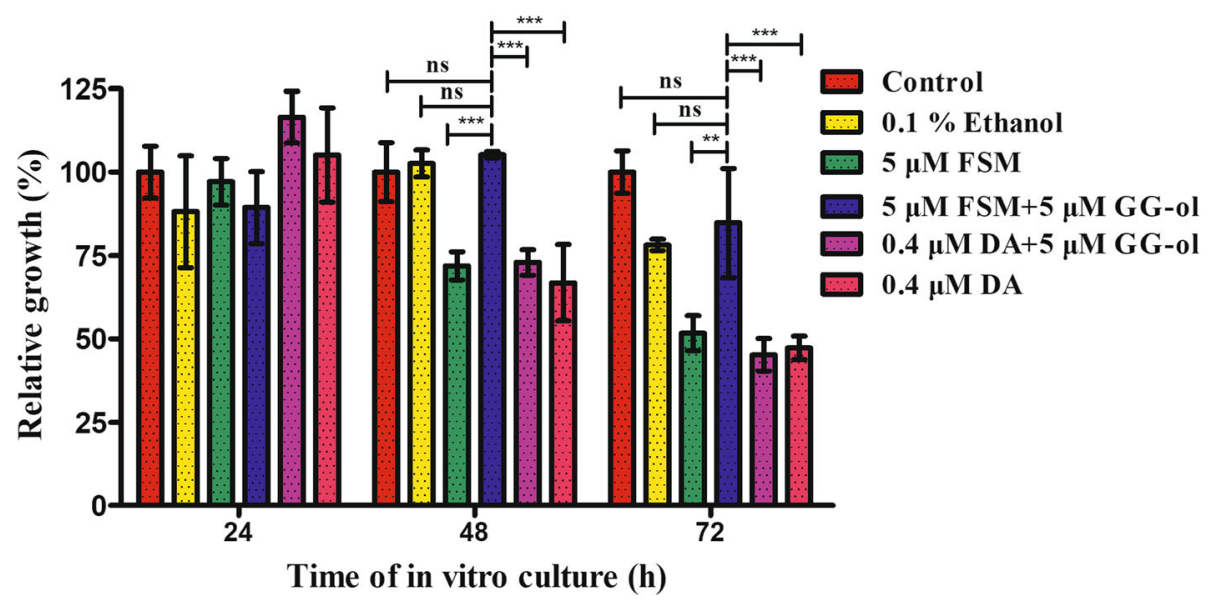

Fig. 6 Efficacy of fosmidomycin on the growth of B. orientalis. In vitro cultured B. orientalis were treated separately with fosmidomycin (FSM), fosmidomycin plus geranylgeraniol (GG-ol), diminazene aceturate (DA), and diminazene aceturate plus geranylgeraniol. The growth of parasites was evaluated every $24 \mathrm{~h}$ for each treatment. $0.1 \%$ ethanol was used as the control. ${ }^{*} P<0.05,{ }^{* *} P<0.01,{ }^{* * *} P<0.001$

Plasmodium falciparum was reported to utilize the non-mevalonate isoprenoid biosynthesis in the apicoplast which is essential to the parasites [8,20,21]. Our previous work demonstrated that $B$. orientalis contains an apicoplast with a $34 \mathrm{kpb}$ circular genome [22], but whether the isoprenoid biosynthesis pathway exists in this parasite remains unknown. In this study, the inhibitory effect of fosmidomycin and the rescue effect of geranylgeraniol on $B$. orientalis indicated the presence of the substrate of this pathway, further suggesting that the MEP pathway is functional and required for the intraerythrocytic growth of $B$. orientalis merozoites, which is similar to that of B. bovis and P. falciparum [15, 21]. The effectiveness of fosmidomycin may be attributed to the presence of a new permeability pathway of erythrocytes, which allows the transport of fosmidomycin across the cell membrane to effectively inhibit the growth of apicomplexan parasites including Plasmodium and Babesia which reside within erythrocytes [19, 23]. The MEP pathway was also reported in Theileria parva, but fosmidomycin showed no inhibitory effect even at a high concentration of $400 \mu \mathrm{M}$ [24].

\section{Conclusions}

In the present study, we cloned and sequenced the seven enzyme genes of the MEP pathway in $B$. orientalis. The native proteins of BoDXS and BoDXR were also identified, which demonstrated that the parasite contains the initial enzyme and the rate-limited enzyme of the MEP pathway. Additionally, the growth of $B$. orientalis could be inhibited by fosmidomycin while the effects of fosmidomycin could be reversed by medium supplemented with geranylgeraniol. These results indicated the existence of the isoprenoid biosynthesis pathway in $B$. orientalis, which could be a potential drug target for screening anti-babesiosis drugs. Further research should focus on the biological function of the MEP pathway in B. orientalis and the screening of new drugs using the $E$. coli model due to its identical isoprenoid biosynthesis pathway with apicomplexan parasites and easiness in manipulation.

\section{Abbreviations}

DMAPP: Dimethylallyl diphosphate; DOXP: 1-deoxy-D-xylulose 5-phosphate; DXR or IspC: 1-deoxy-D-xylulose 5-phosphate reductoisomerase; DXS: 1-deoxyD-xylulose 5-phosphate synthase; IPP: Isopentenyl diphosphate; IspD: 4diphosphocytidyl-2C-methyl-D-erythritol cytidylyltransferase; IspE: 4-diphosphocytidyl2C-methyl-D-erythritol kinase; IspF: 2C-methyl-D-enythritol-2, 4-cyclodiphosphate synthase; IspG: 1-hydroxy-2-methyl-2-(E)-butenyl-4-diphosphate synthase; IspH: 1-hydroxy-2-methyl-2-(E)-butenyl-4-diphosphate reductase; MEP pathway: Methylerythritol phosphate pathway; MEP: 2-C-methyl-D-erythritol 4phosphate (methylerythritol phosphate); MVA: Pathway: mevalonate pathway

\section{Funding}

This work was supported by the National Natural Science Foundation of China (31772729), the National Basic Science Research Program (973 program) of China (2015CB150302), the National Key Research and Development program of China (2017YFD0501201), the Natural Science Foundation of Hubei Province (2017CFA020).

\section{Availability of data and materials}

All data were included as tables and figures within the article. The nucleotide sequences generated during this study were submitted to the GenBank under the accession numbers MH429606-MH429611.

\section{Authors' contributions}

$\mathrm{LH}$ and $\mathrm{JZ}$ designed the study and revised the manuscript. LH wrote the draft of the manuscript. $L H, P H, X L, M L, L Y, J G$ and $X Z$ performed the experiments and analyzed the results. All authors read and approved the final manuscript.

\section{Ethics approval}

This study was approved by the Scientific Ethic Committee of Huazhong Agricultural University (permit number HZAUCA-2017-005). All water buffaloes were handled in accordance with the regulations of the Standing Committee of Hubei People's Congress and the Animal Ethics Procedures and Guidelines of P. R. China. 


\section{Consent for publication}

Not applicable.

\section{Competing interests}

The authors declare that they have no competing interests.

\section{Publisher's Note}

Springer Nature remains neutral with regard to jurisdictional claims in published maps and institutional affiliations.

\section{Author details}

'State Key Laboratory of Agricultural Microbiology, College of Veterinary Medicine, Huazhong Agricultural University, Wuhan 430070, Hubei, People's Republic of China. ${ }^{2}$ Key Laboratory for Development of Veterinary Diagnostic Products, Ministry of Agriculture, Huazhong Agricultural University, Wuhan 430070, Hubei, People's Republic of China. ${ }^{3}$ Key Laboratory of Preventive Veterinary Medicine in Hubei Province, The Cooperative Innovation Center for Sustainable Pig Production, Wuhan 430070, Hubei, China. ${ }^{4}$ Department of Veterinary Pathobiology, College of Veterinary Medicine \& Biomedical Sciences, Texas A\&M University, College Station, Texas, USA.

Received: 8 March 2018 Accepted: 24 July 2018

Published online: 06 August 2018

\section{References}

1. Lau AO, McElwain TF, Brayton KA, Knowles DP, Roalson EH. Babesia bovis: a comprehensive phylogenetic analysis of plastid-encoded genes supports green algal origin of apicoplasts. Exp Parasitol. 2009;123:236-43.

2. Brayton KA, Lau AO, Herndon DR, Hannick L, Kappmeyer LS, Berens SJ, et al. Genome sequence of Babesia bovis and comparative analysis of apicomplexan hemoprotozoa. PLoS Pathog. 2007;3:1401-13.

3. Cornillot E, Hadj-Kaddour K, Dassouli A, Noel B, Ranwez V, Vacherie B, et al. Sequencing of the smallest apicomplexan genome from the human pathogen Babesia microti. Nucleic Acids Res. 2012;40:9102-14

4. Gershenzon J, Dudareva N. The function of terpene natural products in the natural world. Nat Chem Biol. 2007:3:408-14.

5. Bloch K. Sterol molecule: structure, biosynthesis, and function. Steroids. 1992;57:378-83.

6. Hunter WN. Isoprenoid precursor biosynthesis offers potential targets for drug discovery against diseases caused by apicomplexan parasites. Curr Top Med Chem. 2011;11:2048-59.

7. Rohmer M, Knani M, Simonin P, Sutter B, Sahm H. Isoprenoid biosynthesis in bacteria: a novel pathway for the early steps leading to isopentenyl diphosphate. Biochem J. 1993;295:517-24.

8. Jomaa H, Wiesner J, Sanderbrand S, Altincicek B, Weidemeyer C, Hintz M, et al. Inhibitors of the nonmevalonate pathway of isoprenoid biosynthesis as antimalarial drugs. Science. 1999;285:1573-6.

9. Olliaro P, Wells TN. The global portfolio of new antimalarial medicines under development. Clin Pharmacol Ther. 2009:85:584-95.

10. Anthony MP, Burrows JN, Duparc S, Moehrle JJ, Wells TN. The global pipeline of new medicines for the control and elimination of malaria. Malar J. 2012;11:316.

11. He L, Liu Q, Yao B, Zhou Y, Hu M, Fang R, et al. A historical overview of research on Babesia orientalis, a protozoan parasite infecting water buffalo. Front Microbiol. 2017;8:1323.

12. He L, Zhou YQ, Oosthuizen MC, Zhao JL. Loop-mediated isothermal amplification (LAMP) detection of Babesia orientalis in water buffalo (Bubalus babalis Linnaeus, 1758) in China. Vet Parasitol. 2009:165:36-40.

13. Katoh K, Standley DM. A simple method to control over-alignment in the MAFFT multiple sequence alignment program. Bioinformatics. 2016;32: 1933-42.

14. Tamura K, Stecher G, Peterson D, Filipski A, Kumar S. MEGA6: Molecular Evolutionary Genetics Analysis Version 6.0. Mol Biol Evol. 2013;30:2725-9.

15. Caballero MC, Pedroni MJ, Palmer GH, Suarez CE, Davitt C, Lau AO. Characterization of acyl carrier protein and LytB in Babesia bovis apicoplast. Mol Biochem Parasitol. 2012;181:125-33.

16. Imlay L, Odom AR. Isoprenoid metabolism in apicomplexan parasites. Curr Clin Microbiol Rep. 2014;1:37-50.

17. Sauret-Gueto S, Ramos-Valdivia A, Ibanez E, Boronat A, RodriguezConcepcion M. Identification of lethal mutations in Escherichia coli genes encoding enzymes of the methylerythritol phosphate pathway. Biochem Biophys Res Commun. 2003;307:408-15.

18. Kuzuyama T, Shimizu T, Takahashi S, Seto H. Fosmidomycin, a specific inhibitor of 1-deoxy-d-xylulose 5-phosphate reductoisomerase in the nonmevalonate pathway for terpenoid biosynthesis. Tetrahedron Lett. 1998; 39:7913-6.

19. Baumeister S, Wiesner J, Reichenberg A, Hintz M, Bietz S, Harb OS, et al. Fosmidomycin uptake into Plasmodium and Babesia-infected erythrocytes is facilitated by parasite-induced new permeability pathways. PLoS One. 2011; 6:e19334.

20. Odom AR, Van Voorhis WC. Functional genetic analysis of the Plasmodium falciparum deoxyxylulose 5-phosphate reductoisomerase gene. Mol Biochem Parasitol. 2010;170:108-11.

21. Cassera MB, Gozzo FC, D'Alexandri FL, Merino EF, del Portillo HA, Peres VJ, et al. The methylerythritol phosphate pathway is functionally active in all intraerythrocytic stages of Plasmodium falciparum. J Biol Chem. 2004;279: 51749-59.

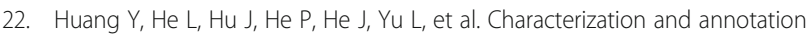
of Babesia orientalis apicoplast genome. Parasit Vectors. 2015;8:543.

23. Kirk K. Membrane transport in the malaria-infected erythrocyte. Physiol Rev. 2001;81:495-537.

24. Lizundia R, Werling D, Langsley G, Ralph SA. Theileria apicoplast as a target for chemotherapy. Antimicrob Agents Chemother. 2009;53:1213-7.
Ready to submit your research? Choose BMC and benefit from:

- fast, convenient online submission

- thorough peer review by experienced researchers in your field

- rapid publication on acceptance

- support for research data, including large and complex data types

- gold Open Access which fosters wider collaboration and increased citations

- maximum visibility for your research: over $100 \mathrm{M}$ website views per year

At $\mathrm{BMC}$, research is always in progress.

Learn more biomedcentral.com/submissions 